\title{
The Realization of Derivation-by-phase Causatives in the Framework of Tree Adjoining Grammar
}

\author{
Qiang Wang \\ College of Foreign Languages, Chongqing University of Posts and Telecommunications, Chongqing, China \\ Email: qiang_wong@ hotmail.com
}

\begin{abstract}
Based on the possibility and necessity of integrating phased-based approach and TAG-based approach, the article attempts to realize the derivation-by-phase causatives in the framework of TAG and, in light of comparison and contrast of four subprograms according to conditions, constraints and hypotheses of the two approaches, finally chooses a subprogram with $v \mathrm{P}_{\mathrm{BE}}$ as the foot node, which is well in line with the Double-Phase Hypothesis proposed in the framework of minimalist syntax, as the one and only acceptable TAG derivation model of causatives.
\end{abstract}

Index Terms - phase theory, tree adjoining grammar, causative, double-phase hypothesis

\section{INTRODUCTION}

Derivation by phase is one of the latest proposals of formal syntax, the Minimalist Program/MP (Chomsky, 2000, $2001,2004,2008$ ) in particular. It aims to carry out syntactic derivation of a construction phase by phase for the purpose of economy principle and efficient computation of the Language Faculty of human beings. Tree Adjoining Grammar/TAG, in computational linguistics, is a grammar formalism originally defined in Joshi (1975). Since then, more variations are developed such as lexicalized TAG. Now the research of tree adjoining grammar is so developed that we have the professional International Workshop on Tree Adjoining Grammar and Related Framework which is held every two years. Up to now, TAG has been used in the formalization of major basic and derived constructions, such as wh-question, relativization, cleft-extraction, raising and equi construction, and passive among others. Since both MP and TAG belong to the formal grammar family, it can be a brilliant idea to study the interactions between them. Frank (2006) made a pioneering attempt in this regard, and uncovered certain thought-provoking relationship there. Causative is a major construction in natural language, so it is necessary to study the derivation of causatives in the framework of TAG, which may provide further insights about the formalized expression of causatives and its application in related fields such as automatic computer processing of causatives and other complex event structures.

This article is composed of four parts. Part I is the introduction. Part II outlines the theoretical framework of phase-based approach and TAG-based approach. Part III, the core part of this article, explores the realization of derivation-by-phase causatives in the framework of TAG of computational linguistics. Part IV summarizes the main findings of this article and provides further problems to be investigated.

\section{Phase-based Approach And TAG-based Approach: The Framework}

\section{A. Phase-based Approach}

Chomsky $(2000,2001,2004)$ claims that all syntactic operations involve a relation between a probe $\mathrm{P}$ and a local goal G which is sufficiently 'close' to the probe. The probe-goal relations must be local in order 'to minimise search', which in turn is forced by the implication that the Language Faculty can only process limited amounts of structure at one time - and, more specifically, can only hold a limited amount of structure in its 'active memory'. In order to ensure a 'reduction of computational burden', Chomsky proposes that 'the derivation of EXP[ressions] proceeds by phase', so that syntactic structures are built up one phase at a time, which should be as small as possible, to minimise memory. More specifically, he suggests that phases are 'propositional' in nature, and include $\mathrm{CP}$ and $v * \mathrm{P}$ (i.e. a $v \mathrm{P}$ with an AGENT or EXPERIENCER external argument). His rationale for taking CP and $v^{*} \mathrm{P}$ as phases is that CP represents a complete clausal complex (including a specification of force), and $v^{*} \mathrm{P}$ represents a complete thematic (argument structure) complex (including an external argument).

Phase theory is explored more explicitly in Chomsky (2004) that they are $\mathrm{CP}$ and $v * \mathrm{P}$, where $\mathrm{C}$ is shorthand for the region that Rizzi (1997) calls the "left periphery," possibly involving feature spread from fewer functional heads; and $v^{*}$ is the functional head associated with full argument structure, transitive and experiencer constructions, and is one of several choices for $v$, which may furthermore be the element determining that the selected root is verbal.

The intuitive idea, to be sharpened, is that features deleted within the cyclic computation remain until the phase level, at which point the whole phase is "handed over" to the phonological component. Hence there is an important condition 
for derivation by phase is Phase Impenetrability Condition/PIC (Chomsky, 2000) as in (1) below.

(1) Phase Impenetrability Condition/PIC: In phase $\alpha$ with head $\mathrm{H}$, the domain of $\mathrm{H}$ is not accessible to operations outside $\alpha$, only $\mathrm{H}$ and its edge are accessible to such operations.

Radford (2004, p. 293) explains the motivation for PIC is that once a complete phase has been formed, the domain of the phase undergoes a transfer operation by which the relevant (domain) structure is simultaneously sent to the phonological component to be assigned an appropriate phonetic representation, and to the semantic component to be assigned an appropriate semantic representation - and from that point on, the relevant domain is no longer accessible to the syntax.

Summarizing the phase theory, Chomsky (2008) further argues that iterated Merge incorporates the effects of three of the EST/Y-model compositional cycles, while eliminating d-and s-structure. Still unaccounted for are the cyclic/compositional mappings to the phonetic and semantic interfaces. These too are incorporated, and the final internal level LF is eliminated, if at various stages of computation there are transfer operations: one hands the syntactic object (SO) already constructed to the phonological component, which maps it to the sensory-motor (SM) interface ("Spell-Out"); the other hands SO to the semantic component, which maps it to the conceptual-intentional (C-I) interface. These SOs are called phases. For more information about phase theory, see He (2007) and Tang (2009).

Above is the framework upon which we develop our double-phase hypothesis for syntactic derivation of causatives. We may find that there are two $\mathrm{V}^{*} \mathrm{Ps}$ in the syntax of causatives, i.e., two phases, and that PIC is perfectly observed in the hypothesis. A further step is to show how to realize such derivation in the framework of TAG which to be discussed in Part III.

\section{B. TAG-based Approach}

Tree adjoining grammar initiated by Joshi (1975) takes tree as the elementary unit of rewriting and has rules for rewriting the nodes of trees as other trees. There are two types of basic trees in TAG: initial trees (" $\alpha$ ") and auxiliary trees (" $\beta$ "). A derivation starts with an initial tree, combining via either substitution or adjunction. Substitution replaces a frontier node with another tree whose top node has the same label. Adjunction inserts an auxiliary tree into the center of another tree. Substitution and adjunction are schematized as below (see Frank, 2006).
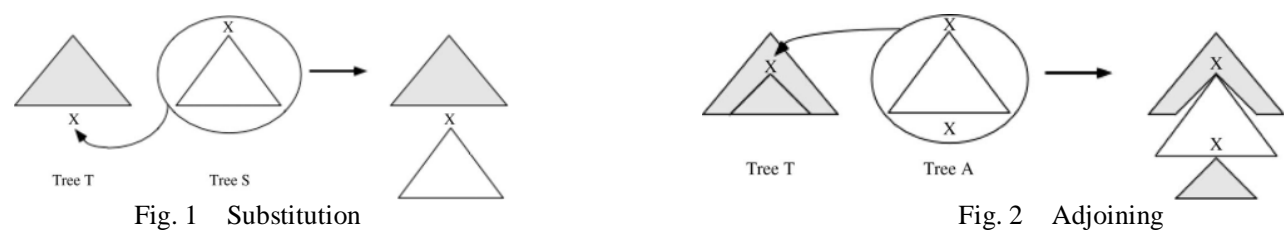

Kroch \& Joshi (1985) states that a TAG defines a finite set of simple sentence elementary trees and an adjunction operation that produces complex sentences through the combination of simple sentences. What TAG contributes to the theory of grammar is a constrained theory of syntactic embedding, one requiring that embedded structures be composed out of elementary structures in a fixed way and one which forces co-occurrence relations between elements that are separated in surface constituent structure to be stated locally as constraints on elementary trees in which those elements are co-present. It is also pointed out that the elementary trees (initial and auxiliary trees) are the appropriate domains for characterizing certain dependencies (e.g., subcategorization dependencies and filler-gap dependencies). Each step in the derivation selects an elementary tree together with a set of appropriate lexical items. Now that as we select the lexical items for each elementary tree we can check a variety of constraints on the set of lexical items. Also, Kroch \& Joshi (1985) present in important constraint on TAG as in (2) below.

(2) Null adjoining local constraint: No auxiliary tree is adjoinable at the node $n$.

This implies that TAG disallows rightward derivation. Since it is so easy and obvious to identify the direction of a derivation, it will be firstly used to judge whether a TAG derivation is grammatical or not later.

Morawietz \& Mönnich (2001) sketches a definition of tree adjoining grammar with a strong flavor of formalism as in (3) below.

(3) A Tree Adjoining Grammar (TAG) is a quintuple $\left\langle V_{N}, V_{T}, S, I, A>\right.$ where $V_{N}$ is a finite set of nonterminals, $V_{T}$ a finite set of terminals, $S \in V_{N}$ the start symbol, I a finite set of initial trees and A a finite set of auxiliary trees.

According to them, initial trees are such that all interior nodes (including the root node) are labeled with nonterminals and all nodes on the frontier are labeled with terminal or nonterminal symbols; the nonterminals being marked for substitution. The same holds for the auxiliary trees with one exception. There exists one distinguished leaf-node which is labeled with the same nonterminal as the root node, which is called the foot node. New trees are built from the sets $I$ and $A$ via adjunction or substitution. Adjunction is defined such that an auxiliary tree is spliced into an existing tree such that it basically "expands" a nonterminal. A subtree rooted in the node labeled with a nonterminal $A$ is taken out of a tree. A new auxiliary tree is inserted in its place (if the root and foot are also labeled with the identical nonterminal $A$ ) and the original subtree is appended at the foot node. There also exists a simpler operation in TAGs, called substitution, to generate new trees. Intuitively, in substitution, a nonterminal is replaced by a tree with a matching nonterminal at its root. Evidently, these are in line with the original framework of Joshi (1975). For more information, see Weng \& Wang 
(1998) and $\mathrm{Yu}(2003)$.

\section{Possibility and Necessity of Integrating Phase-based Approach and TAG-based Approach}

Generally speaking, both phase-based approach and TAG-based approach belong to context-free grammar. Since so, it's possible and necessary to integrate them in solving specific linguistic issues. But how?

Emphasis on locality is one of the spirits of the TAG formalism and those of phase theory as well. The constraints on lexical insertion are always local, so the TAG formalism treats insertion locally. On such very basis, it is reasonable to integrate phase theory and tree adjoining grammar. In this regard, Frank (2005) made breakthroughs, where a series of difference between the notion of elementary tree structure and that of phase were explored. First, the lexical array that underlies the construction of an elementary tree is somewhat larger than that which feeds a phase as that notion is usually understood. In particular, the Condition on Elementary Tree Minimality requires the heads in an elementary tree must form part of the extended projection of a single lexical head. As a result, the lexical array underlying an elementary tree must include a lexical head and may include any number of functional heads that are associated with that lexical head.

Also in Frank (2006), it is argued that the phase-based approach to grammatical derivation and one rooted in the TAG formalism share the idea that the derivation of a complex sentence is divided into separate derivations of local domains. However, the TAG-based approach to syntactic derivations allows us to derive island-based locality from the derivational architecture, which stands in contrast to the phase-based approach, where adjuncts should be transparent to extraction unless further stipulations are made.

In addition to the common feature of locality between the two approaches, they share the same basic operations, that is, substitution and adjucntion. In the framework of minimalist syntax, Chomsky (1995) takes substitution and adjucntion as the basic syntactic operations, that are revised to "set Merge" and "pair Merge" respectively in Chomsky (2000), and to "Merge" collectively in Chomsky (2001, 2004, 2008). Chomsky (1995, p. 248-249) stipulates that, suppose there are two objects $\alpha$ and $\beta$, substitution forms $L=\{H(K),\{\alpha, K\}\}$, where $H(K)$ is the head (=the label) of the projected element $K$. But when $\alpha$ adjoins to $K$ and the target projects, adjunction takes $L=\{<H(K), H(K)>,\{\alpha, K\}\}$ in which $\langle\mathrm{H}(\mathrm{K}), \mathrm{H}(\mathrm{K})\rangle$, the label of $\mathrm{L}$, is not a term of the structure formed. It is not identical to the head of $\mathrm{K}$ as before though it is constructed from it in a trivial way. Therefore, adjunction differs from substitution only in that adjunction forms a two-segment category rather than a new category. We may easily note that the operation processes of substitution and adjunction are extremely similar to those in the framework of TAG. In addition, Merge and adjunction in syntax always applies in the simplest possible form: at the root which is also the place that adjunction operates in the framework of TAG as is shown in Fig. 1 and 2 above. All of these lay a solid foundation for the integration of the two approaches.

Frank (2006) presents a fundamental TAG hypothesis as in (4) below.

(4) The fundamental TAG hypothesis: Every syntactic dependency is expressed locally within a single elementary tree.

This hypothesis substantially constrains the nature of elementary trees: they must be large enough to express all syntactic dependencies. At the same time, if our goal is to derive locality conditions from properties of the TAG derivation, these trees must not be too large. With sufficiently large elementary trees, a dependency within a single elementary tree could violate locality conditions, and the TAG system of derivation will say nothing about such violations.

Frank (2006) points out that when a complex structure is built in a TAG derivation, we can always view it as deriving from a single elementary tree into which other trees have adjoined or substituted. Such TAG derivations must be constrained by a Markovian condition as in (5) below.

(5) Markovian condition on TAG derivations: Each step in a TAG derivation must involve the wellformed combination of two elementary trees.

What the Markovian condition requires is that the combination of two complex trees $\alpha$ and $\beta$ cannot depend on the presence of any structure that has been adjoined or substituted during the construction of $\alpha$ and $\beta$, but only on the properties of the two elementary trees that are the "core" of the derivations of $\alpha$ and $\beta$.

As a nice interface between phase-based approach, syntactic tree in particular, and TAG-based approach, a condition on elementary tree minimality is also made in Frank (2006) as in (6) below.

(6) Condition on elementray tree minimality: The heads in an elementary tree must form part of the extended projection of a single lexical head.

As a result, the lexical array underlying an elementary tree must include a lexical head and may include any number of functional heads that are associated with that lexical head.

If any proposal attempts to integrate both phase-based approach and TAG-based approach, the operations must be substitution and adjunction while all the constraints specified in both approaches must be observed, including the locality principle (i.e. PIC), the Markovian condition on TAG, and the Condition on Elementary Tree Minimality. These are also the requirements for our proposal to be stated in Part III below.

\section{The Realization of Derivation-By-phase Causatives in the Framework of TAG}




\section{A. Syntactic Derivation of Causatives by Phase}

In the minimalist syntax, Wang (2009a, 2009b) gives up the proposal of derivation between periphrastic causative construction and lexical causative construction, inherits the proposal of non-derivation between them on a critical basis, and points out that causatives are affected applicatives in nature. As a new proposal of syntactic derivation of causatives, a Double-Phase Hypothesis of Causatives is proposed that there are two phases, i.e. VoiceP and $v \mathrm{P}_{\mathrm{BE}}$, in causatives, which contains two functional heads, i.e. CAUSE and AFFECTED, respectively. In the $v \mathrm{P}_{\mathrm{BE}}$ phase, AFFECTED selects the verbal V as its complement, and after Merge, further selects direct internal argument as its Spec; in the VoiceP phase, $v_{\mathrm{DO}}$ selects $v \mathrm{P}_{\mathrm{BE}}$ as its complement, and after Merge, $v \mathrm{P}_{\mathrm{DO}}$ is in turn selected by CAUSE/Voice as its complement while CAUSE/Voice further selects external causer argument as its Spec. The derivation process is shown in (7) below.

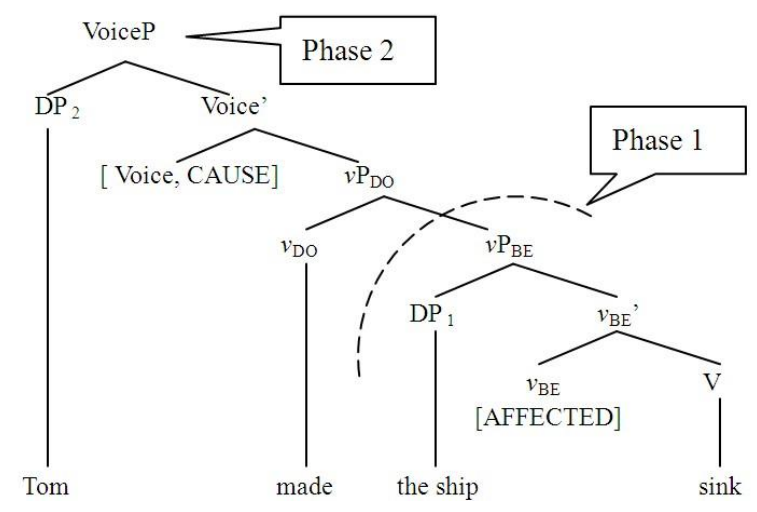

Obviously, VoiceP and $v \mathrm{P}_{\mathrm{BE}}$ are both typical $v \mathrm{Ps}$ with external arguments, hence are phases. In addition, such double-phase derivation of causatives perfectly observes the PIC, and also the hypothesis that there are two functional heads, i.e., CAUSE and AFFECTED, in causatives, matches the intuition that there are cause event and effect event in causatives. In light of this, we hold that the Double-Phase Hypothesis of Causatives is a rational proposal for the syntactic derivation of causatives. Next our task is to realize this derivation program in the framework of TAG.

\section{B. Derivation-by-phase Causatives in the Framework of TAG}

Based on the stipulations of TAG, there can be several possible programs for the derivation of causatives. Program 1 can be made that the derivation is accomplished in one stroke and that there is one initial tree yet without any auxiliary tree. Hence there should be no adjunction or substitution here. Such TAG derivation model is rather similar to the syntactic derivation as shown in (7) above at the first sight except that no phases are marked any more. The TAG derivation model of Program 1 is shown in (8) below.

(8) Program 1: only one initial tree, no auxiliary tree and no adjunction

Initial tree $\alpha_{0}$

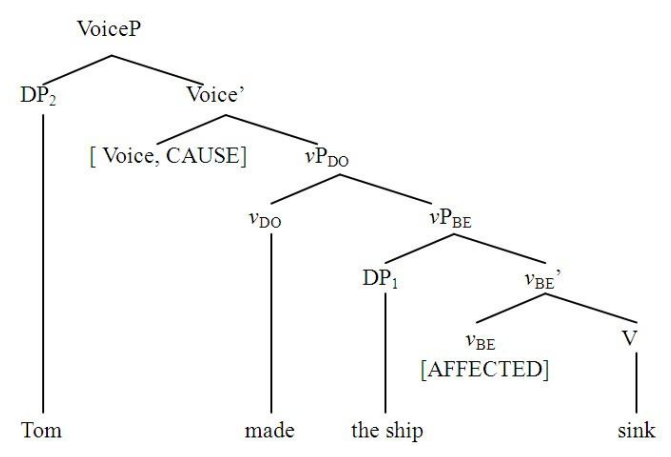

However, Program 1 in which only one elementary tree is involved, is in violation of the Markovian condition on TAG derivations as described in (5) above, which requires that each step in a TAG derivation involve the well-formed combination of two elementary trees. Moreover, since our goal is to derive locality conditions from properties of the TAG derivation, the elementary trees must not be too large, according to the fundamental TAG hypothesis as described in (4) above. But now the elementary tree in Program 1 is so large that a dependency within a single elementary tree could violate locality conditions, and the TAG system of derivation will say nothing about such violations. So, Program 1 must not be taken as a reasonable program for derivation of causatives in the framework of TAG.

Program 2 can be made that there is one initial tree and one auxiliary tree. And three subprograms can be further made as below in which the structure is parsed after "Tom", "made" and "the ship" respectively.

(9) Subprogram 1: adjoin $\beta_{1}$ to $\alpha_{1}$ 
Initial tree $\alpha_{1}$ (where $v \mathrm{P}_{\mathrm{DO}}$ is the foot node)

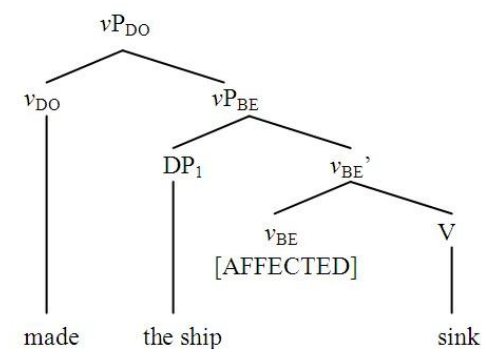

(10) Subprogram 2: adjoin $\beta_{2}$ to $\alpha_{2}$

Initial tree $\alpha_{2}$ (where $v \mathrm{P}_{\mathrm{BE}}$ is the foot node)

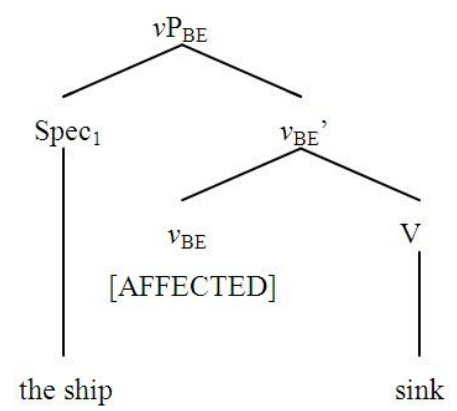

(11) Subprogram 3: adjoin $\beta_{3}$ to $\alpha_{3}$

Initial tree $\alpha_{3}$ (where $\mathrm{V}$ is the foot node)

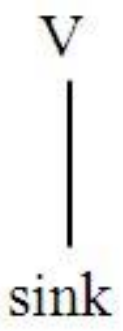

(12) Subprogram 4: adjoin $\beta_{4}$ to $\alpha_{4}$ Initial tree $\alpha_{4}$ (where $v_{\text {DO }}$ is the foot node)

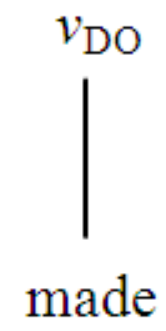

Auxiliary tree $\beta_{1}$

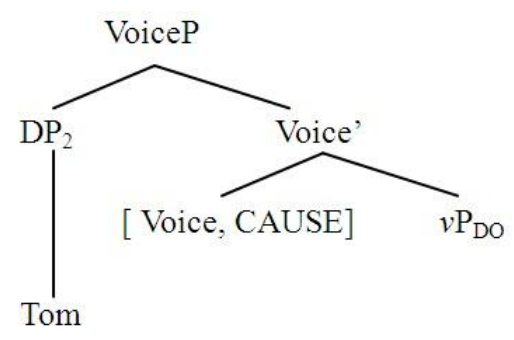

Auxiliary tree $\beta_{2}$

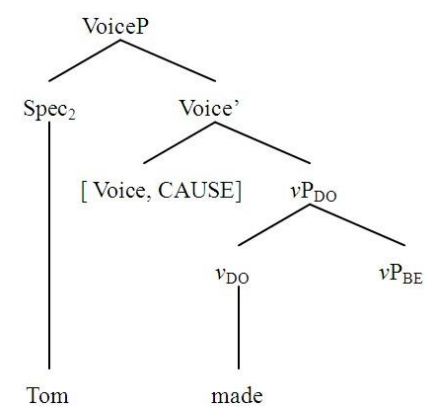

Auxiliary tree $\beta_{3}$

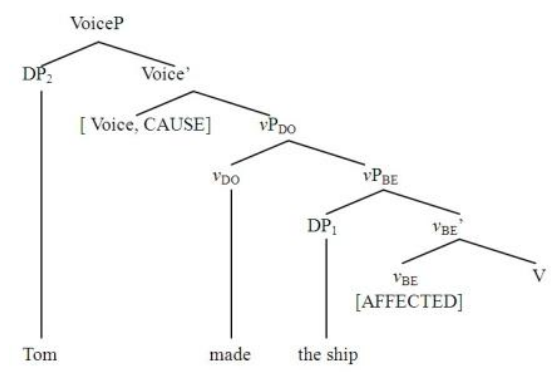

Auxiliary tree $\beta_{4}$

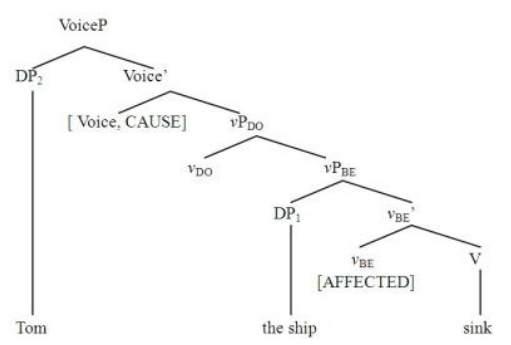

Given such constraints as PIC, Markovian condition on TAG hypothesis, condition on elementray tree minimality, and null adjoining local constraint among others, which possible subprogram above is acceptable in the framework of TAG?

First, according to what is implied in the null adjoining local constraint, that is, only leftward derivation is allowed, it can be safely said that subprogram 1-4 are all grammatical as their derivations are all leftward.

Next, we confirm the grammaticalness of the four subprograms mainly according to the fundamental TAG hypothesis, which requires that the elementary tree must be large enough but not too large. Locality must be respected there. It can be seen that the auxiliary tree $\beta_{1}$, the initial tree $\alpha_{3}$, and the initial tree $\alpha_{4}$, are all so small that syntactic dependencies are not perfectly expressed there, whereas correspondingly the initial tree $\alpha_{1}$, the auxiliary tree $\beta_{3}$, and the auxiliary tree $\beta_{4}$, are all so large that TAG can say nothing about its locality and other properties. In contrast, the initial tree $\alpha_{2}$ and the auxiliary tree $\beta_{2}$ are neither too large nor too small, where all syntactic dependencies are expressed well 
and meanwhile locality conditions are respected well. Therefore, among the four subprograms, only the subprogram 2 complies with the fundamental TAG hypothesis. More importantly, the subprogram 2 also complies with the basic proposal of phase theory that a structure is derived phase by phase and the specific Double-Phase Hypothesis of Causatives. This shows the effect of common features between phase-based approach and TAG-based approach.

Then, it can be found that there is at least one lexical head in the initial trees and auxiliary trees in the four subprograms while the two functional heads, i.e. CAUSE and AFFECTED, appear optionally any lexical insertion in some trees without. So, the condition on elementray tree minimality can not be used here to exclude any subprogram above.

Up to now, we have considered the derivation of causatives in the framework of TAG according to its conditions, constraints or hypothesis. It can be safely concluded that the subprogram 2, the derivation model maded based on our Double-Phase Hypothesis of Causatives, is the one and only acceptable program in the framework of TAG.

\section{CONCLUSION}

The article outlines the basic proposals of phased-based approach and TAG-based approach, presents the possibility and necessity of integrating the two approaches, proposes programs to derive causatives in the framework of TAG among which the subprogram with $\nu \mathrm{P}_{\mathrm{BE}}$ as the foot node is chosen as the one and only acceptable derivation model of causatives. Luckily, the subprogram chosen is also in line with the Double-Phase Hypothesis proposed in the framework of minimalist syntax. This research is helpful for us to know more about the structure of causatives and its application in the field of computational linguistics.

\section{ACKNOWLEDGEMENT}

This research is sponsored by "Building an English Major with IT Characteristics against the Background of Globalization", a major promotion project of Chongqing University of Posts and Telecommunications 2008, and "A Study of Interface between Syntax and Computational Linguistics", a planned project of philosophy and social science of Chongqing Municipality 2009 (Grant No.: SKZ0909).

\section{REFERENCES}

[1] Chomsky, N. (1995). The Minimalist Program. Cambridge, MA.: The MIT Press.

[2] Chomsky, N. (2000). Minimalist inquiries: the framework. In: Martin, R., D. Michaels \& J. Uriagereka. (eds.), Step by Step, Essays on Minimalist Syntax in Honor of Howard Lasnik. MIT Press, Cambridge, MA, pp. 89-155.

[3] Chomsky, N. (2001). Derivation by phase. In: Kenstowicz, M. (ed.), Ken Hale: a life in language. MIT Press, Cambridge, MA, pp. 1-52.

[4] Chomsky, N. (2004). Beyond explanatory adequacy. In: Belletti, A. (ed.), Structures and Beyond, The Cartography of Syntactic Strutures, Volume 3. Oxford University Press, New York, pp. 104-131.

[5] Chomsky, N. (2008). On phases. In Freidin, R., C. Otero \& M. Zubizarreta. (eds.), Foundational issues in linguistic theory. Cambridge, MA: The MIT Press, pp. 133-166.

[6] He, X. (2007). Phases and their syntactic derivation: Recent development in Chomskyan syntactic theory, Foreign Language Teaching and Research, 39, 345-351

[7] Joshi, A. (1975). Tree Adjunct Grammars. Journal of Computer and System Sciences, 10, 136-163.

[8] Kroch, A. \& A. Joshi. (1985). The Linguistic Relevance of Tree Adjoining Grammar. Technical report, MS-CIS-85-16. Department of Computer and Information Science, University of Pennsylvania.

[9] Morawietz, F. \& U. Mönnich. (2001). A Model-Theoretic Description of Tree Adjoining Grammars. Electronic Notes in Theoretical Computer Science, 53, 210-232.

[10] Radford, A. (2004). Minimalist Syntax: Exploring the Structure of Syntax. Cambridge: Cambridge University Press.

[11] Rizzi, L. (1997). The fine structure of the left periphery. In Haegeman, L. (ed.), Elements of Grammar, Kluwer, Dordrecht, pp. 281-337.

[12] Robert F. (2006). Phase theory and Tree Adjoining Grammar. Lingua, 116, 145-202.

[13] Tang, S. (2009). Phasehood in Syntactic Derivations: An Introductory Survey. Contemporary Linguistics, 11, 207-215.

[14] Wang, Q. (2009a). On the Double-Phase Hypothesis of English and Chinese Causatives. Modern Foreign Languages, 32 , 331-341.

[15] Wang, Q. (2009b). On Causation, Causativized Verb and Causative Construction. Tianjin Foreign Studies University Journal, $16,19-29$.

[16] Weng, F. \& Y. Wang. (1998). Introduction of Computational Linguistics. Beijing: China Social Sciences Press.

[17] Yu, S. (2003). Computational Linguistics: An Introduction. Beijing: The Commercial Press.

Qiang Wang was born in Hunan, China in 1979. He received his MA degree in linguistics from Sichuan International Studies University, China in 2007.

$\mathrm{He}$ is currently a lecturer in the College of Foreign Languages, Chongqing University of Posts and Telecommunications in southwest China. His research interests include syntax, biolinguistics and computational linguistics. 